PROCEEDINGS OF THE

AMERICAN MATHEMATICAL SOCIETY

Volume 139, Number 3, March 2011, Pages 1121-1126

S 0002-9939(2010)10673-1

Article electronically published on September 30, 2010

\title{
CHARACTERIZATION OF SUBDIAGONAL ALGEBRAS
}

\author{
TURDEBEK N. BEKJAN
}

(Communicated by Marius Junge)

\begin{abstract}
Let $\mathcal{M}$ be a finite von Neumann algebra with a faithful normal tracial state $\tau$, and let $\mathcal{A}$ be a tracial subalgebra of $\mathcal{M}$. We show that $\mathcal{A}$ has $L^{p}$-factorization $(1 \leq p<\infty)$ if and only if $\mathcal{A}$ is a subdiagonal algebra. Also, we obtain some characterizations of subdiagonal algebras.
\end{abstract}

\section{INTRODUCTION}

Let $\mathcal{M}$ be a finite von Neumann algebra with a faithful normal tracial state $\tau$. In [1, Arveson introduced the notion of finite, maximal, subdiagonal algebras $\mathcal{A}$ of $\mathcal{M}$, as noncommutative analogues of weak-* Dirichlet algebras. Subsequently several authors studied the (noncommutative) $\mathcal{H}^{p}$-spaces associated with such algebras ([9, 11, 12, 13, 14, 16, 17, 18]). Arveson 11 proved a Szegö type factorization theorem. Some extensions can be found in [8, 18, and [15]. Labuschagne [10] proved a non-commutative version of Szegö's theorem. In the recent arcticles [3, 4, 5], among other things, Blecher and Labuschagne studied tracial subalgebras of $\mathcal{M}$ and gave several characterizations of subdiagonal algebras. They proved that if a tracial subalgebra $\mathcal{A}$ has $L^{\infty}$-factorization, then $\mathcal{A}$ is a subdiagonal algebra. We will consider the $L^{p}$-factorization $(0<p<\infty)$ property of tracial subalgebras. This paper is organized as follows. Section 1 contains some preliminary definitions. In section 2 , we prove that if a tracial subalgebra $\mathcal{A}$ has $L^{p}$-factorization $(1 \leq p<\infty)$, then $\mathcal{A}$ is a subdiagonal algebra. In section 3 , we consider tracial subalgebras, which satisfy $L^{2}$-density. We show that if a tracial subalgebra $\mathcal{A}$ has $L^{p}$-factorization $(0<p<1)$ and satisfies $L^{2}$-density, then $\mathcal{A}$ is a subdiagonal algebra.

\section{Preliminaries}

Throughout this paper, we denote by $\mathcal{M}$ a finite von Neumann algebra on a Hilbert space $\mathcal{H}$ with a faithful normal tracial state $\tau$. For $0<p<\infty$ we denote by $L^{p}(\mathcal{M})$ the usual noncommutative $L^{p}$-space associated with $(\mathcal{M}, \tau)$. Recall that $L^{\infty}(\mathcal{M})=\mathcal{M}$, equipped with the operator norm. It is well-known that $L^{p}(\mathcal{M})$ is a Banach space under $\|\cdot\|_{p}(1 \leq p \leq \infty)$ satisfying all the expected properties such as duality of $L^{p}(\mathcal{M})$ and $L^{q}(\mathcal{M})$ for $\frac{1}{p}+\frac{1}{q}=1$ (see [7, 20]).

Received by the editors November 30, 2009 and, in revised form, April 13, 2010.

2010 Mathematics Subject Classification. Primary 46L51, 46L52, 47L75.

Key words and phrases. von Neumann algebras, tracial subalgebra, subdiagonal algebra, $L^{2}$-density, $L^{p}$-factorization.

The author was partially supported by NSFC grant No. 10761009 .

(C)2010 American Mathematical Society Reverts to public domain 28 years from publication 
For a subset $K$ of $L^{p}(\mathcal{M})$, put $J(K)=\left\{x^{*}: x \in K\right\}, K^{-1}=\left\{x: x, x^{-1} \in K\right\}$, $K^{+}=\{x: x \geq 0, x \in K\}$, and $[K]_{p}$ the closed linear span of $K$ in $L^{p}(\mathcal{M})$. (Here $[K]_{\infty}$ is the weak* closure of $K$.)

Given a von Neumann subalgebra $\mathcal{N}$ of $\mathcal{M}$, an expectation $\mathcal{E}: \mathcal{M} \rightarrow \mathcal{N}$ is defined to be a positive linear map which preserves the identity and satisfies $\mathcal{E}(x y)=x \mathcal{E}(y)$ for all $x \in \mathcal{N}$ and $y \in \mathcal{M}$. Since $\mathcal{E}$ is positive it is Hermitian; i.e. $\mathcal{E}(x)^{*}=\mathcal{E}\left(x^{*}\right)$ for all $x \in \mathcal{M}$. Hence $\mathcal{E}(y x)=\mathcal{E}(y) x$ for all $x \in \mathcal{N}$ and $y \in \mathcal{M}$. For a complete study of $\mathcal{E}$, we refer to [1, 12.

Definition 1.1. Let $\mathcal{A}$ be a $\mathrm{w}^{*}$-closed unital subalgebra of $\mathcal{M}$. If there exists a linear projection $\mathcal{E}$ from $\mathcal{A}$ onto $\mathcal{D}=\mathcal{A} \cap J(\mathcal{A})$ such that

(i) $\mathcal{E}(x y)=\mathcal{E}(x) \mathcal{E}(y), \forall x, y \in \mathcal{A}$;

(ii) $\tau \circ \mathcal{E}=\tau$,

then $\mathcal{A}$ is called a tracial subalgebra of $\mathcal{M}$.

Definition 1.2. Let $\mathcal{A}$ be a $\mathrm{w}^{*}$-closed unital subalgebra of $\mathcal{M}$, and let $\mathcal{E}$ be a faithful, normal expectation from $\mathcal{M}$ onto the diagonal von Neumann algebra $\mathcal{D}=$ $\mathcal{A} \cap J(\mathcal{A})$. Then $\mathcal{A}$ is a finite subdiagonal subalgebra of $\mathcal{M}$ with respect to $\mathcal{E}$ if:

(i) $\mathcal{A}+J(\mathcal{A})$ is $\mathrm{w}^{*}$-dense in $\mathcal{M}$;

(ii) $\mathcal{E}(x y)=\mathcal{E}(x) \mathcal{E}(y), \forall x, y \in \mathcal{A}$;

(iii) $\tau \circ \mathcal{E}=\tau$.

By Theorem 5.6 of $[3, \mathcal{E}$ is precisely the restriction to $\mathcal{A}$ of the unique faithful normal conditional expectation $\Phi$ from $\mathcal{M}$ onto $\mathcal{D}$ such that $\tau=\tau \circ \Phi$. Hence we may continue to write $\Phi$ as $\mathcal{E}$, and we call this extension the conditional expectation onto $\mathcal{D}$. It is well-known that $\mathcal{E}$ extends to a contractive projection from $L_{p}(\mathcal{M})$ onto $L_{p}(\mathcal{D})$ for every $1 \leq p \leq \infty$. The extension will still be denoted by $\mathcal{E}$.

Let $\mathcal{A}_{0}=\mathcal{A} \cap \operatorname{ker}(\mathcal{E})$. We say that $\mathcal{A}$ is $\tau$-maximal if

$$
\mathcal{A}=\left\{x \in \mathcal{M}: \tau(x y)=0, \forall y \in \mathcal{A}_{0}\right\} .
$$

\section{FACTORIZATION}

Let $\mathcal{A}$ be a tracial subalgebra of $\mathcal{M}$. For $0<p<\infty$, we write $\mathcal{A}_{p}$ for $[\mathcal{A}]_{p} \cap \mathcal{M}$.

Lemma 2.1. Let $\mathcal{A}$ be a tracial subalgebra of $\mathcal{M}$. Then $\mathcal{A}_{p}$ is a tracial subalgebra of $\mathcal{M}$ for $1 \leq p<\infty$.

Proof. The proof is the same as that of Theorem 4.4 in [4]. We only need to prove the following:

$$
\mathcal{E}(a b)=\mathcal{E}(a) \mathcal{E}(b), \forall a, b \in \mathcal{A}_{p} .
$$

Indeed, let $b \in \mathcal{A}_{p}$. Then there exists a sequence $\left\{b_{n}\right\}$ in $\mathcal{A}$ such that $b_{n} \rightarrow b$ in $L_{p}(\mathcal{M})$. So for all $a \in \mathcal{A}$ we have $a b_{n} \in \mathcal{A}$, and $a b_{n} \rightarrow a b$ in $L_{p}(M)$. Thus

$$
\mathcal{E}(a b)=\lim _{n \rightarrow \infty} \mathcal{E}\left(a b_{n}\right)=\lim _{n \rightarrow \infty} \mathcal{E}(a) \mathcal{E}\left(b_{n}\right)=\mathcal{E}(a) \mathcal{E}(b) .
$$

Hence, by what we just proved,

$$
\mathcal{E}(a b)=\lim _{n \rightarrow \infty} \mathcal{E}\left(a b_{n}\right)=\lim _{n \rightarrow \infty} \mathcal{E}(a) \mathcal{E}\left(b_{n}\right)=\mathcal{E}(a) \mathcal{E}(b), \forall a \in \mathcal{A}_{p} .
$$

Definition 2.2. Let $0<p \leq \infty$, and let $\mathcal{A}$ be a tracial subalgebra of $\mathcal{M}$. We say that $\mathcal{A}$ has $L^{p}$-factorization, if for all $x \in L^{p}(\mathcal{M})^{-1}$, there is a unitary $u \in \mathcal{M}$ and $a \in[\mathcal{A}]_{p}^{-1}$ such that $x=u a$. 
Proposition 2.3. Let $\mathcal{A}$ be a tracial subalgebra of $\mathcal{M}$. Consider the following conditions:

(i) For some $1 \leq p_{0}<\infty, \mathcal{A}$ has $L^{p_{0}}$-factorization.

(ii) For some $0<p_{0}<1, \mathcal{A}$ has $L^{p_{0}}$-factorization and $\mathcal{A}_{p_{0}}$ is a tracial subalgebra of $\mathcal{M}$.

Then either one of the conditions (i) or (ii) implies that $\mathcal{A}$ has $L^{p}$-factorization for all $0<p<p_{0}$.

Proof. Let $x \in \mathcal{M}^{-1} \subset L^{p_{0}}(\mathcal{M})^{-1}$. Then there is a unitary $u \in \mathcal{M}$ and $a \in[\mathcal{A}]_{p_{0}}^{-1}$ such that $x=u a$, since $\mathcal{A}$ has $L^{p_{0}}$-factorization. So $a \in \mathcal{A}_{p_{0}}^{-1}$, and therefore $\mathcal{A}_{p_{0}}$ has $L^{\infty}$-factorization. By Theorem 1.1 of [4, $\mathcal{A}_{p_{0}}$ is a subdiagonal subalgebra of $\mathcal{M}$. Let $x \in L^{p}(\mathcal{M})^{-1}$. By Theorem 3.1 of [2], there is a unitary $u \in \mathcal{M}$ and $a \in\left[\mathcal{A}_{p_{0}}\right]_{p}^{-1}$ such that $x=u a$. On the other hand, we have

$$
[\mathcal{A}]_{p} \subset\left[\mathcal{A}_{p_{0}}\right]_{p} \subset\left[[\mathcal{A}]_{p_{0}}\right]_{p}=[\mathcal{A}]_{p} .
$$

Thus $a \in[\mathcal{A}]_{p}^{-1}$, and so $\mathcal{A}$ has $L^{p}$-factorization.

Theorem 2.4. Let $\mathcal{A}$ be a tracial subalgebra of $\mathcal{M}$. Then the following conditions are equivalent:

(i) $\mathcal{A}$ is a subdiagonal subalgebra of $\mathcal{M}$.

(ii) For all $0<p \leq \infty, \mathcal{A}$ has $L^{p}$-factorization.

(iii) For some $1 \leq p \leq \infty, \mathcal{A}$ has $L^{p}$-factorization.

Proof. (i) $\Rightarrow$ (ii) follows by Theorem 3.1 of [2] (also see Theorem 4.2.1 of [1], Corollary 4.13 of [5] and the remark following Theorem 8.1 of [15]).

(ii) $\Rightarrow$ (iii) is clear.

(iii) $\Rightarrow$ (i). When $p=\infty$, it is (b) of Theorem 1.1 of [4]. From the proof of Proposition 2.3, we know that $\mathcal{A}_{p}$ is a subdiagonal subalgebra of $\mathcal{M}$. We claim that $\mathcal{A}_{p}=\mathcal{A}$. Indeed, it suffices to show that if $y \in L^{1}(\mathcal{M})$ and $\tau(y a)=0$ for each $a \in \mathcal{A}$, then $\tau(y a)=0$ for each $a \in \mathcal{A}_{p}$. Let $y=v|y|$ be the polar decomposition of $y$, and $v_{1} \in \mathcal{M}$ such that $|y|=v_{1}(|y|+I)$. Notice that $\mathcal{A}_{p}$ is a subdiagonal subalgebra and $|y|+I \in L^{1}(\mathcal{M}),(|y|+I)^{-1} \in \mathcal{M}$. Hence, there is a unitary $u \in \mathcal{M}$ and $b \in\left[\mathcal{A}_{p}\right]_{1}$ such that $|y|+I=u b, b^{-1} \in \mathcal{A}_{p}$. So $y=w b$, where $w=v v_{1} u \in \mathcal{M}$. On the other hand,

$$
[\mathcal{A}]_{1}=\left[b^{-1} b \mathcal{A}\right]_{1} \subset\left[b^{-1}\left[\mathcal{A}_{p}\right]_{1}\right]_{1} \subset\left[\mathcal{A}_{p}\right]_{1}=[\mathcal{A}]_{1} .
$$

Since $\tau(w b a)=\tau(y a)=0$ for each $a \in \mathcal{A}$, it follows that $\tau(w a)=0$ for each $a \in\left[\mathcal{A}_{p}\right]_{1}$. By $\tau$-maximality of $\mathcal{A}_{p}$ we obtain $w \in\left(\mathcal{A}_{p}\right)_{0}$. Hence $y \in\left[\left(\mathcal{A}_{p}\right)_{0}\right]_{1}$, or $\tau(y a)=0$ for each $a \in \mathcal{A}_{p}$.

Theorem 2.5. Let $\mathcal{A}$ be a $\tau$-maximal tracial subalgebra of $\mathcal{M}$. Then the following conditions are equivalent:

(i) $\mathcal{A}$ is a subdiagonal subalgebra of $\mathcal{M}$.

(ii) For some $0<p<1, \mathcal{A}$ has $L^{p}$-factorization and $\mathcal{A}_{p}$ is a tracial subalgebra of $\mathcal{M}$.

Proof. We need only prove (ii) $\Rightarrow(\mathrm{i})$. It is clear that $\mathcal{A}_{p}$ is a subdiagonal subalgebra of $\mathcal{M}$ and $\mathcal{A} \subset \mathcal{A}_{p}$. If $b \in \mathcal{A}_{p}$, then $\tau(b a)=\tau(\mathcal{E}(b a))=\tau(\mathcal{E}(b) \mathcal{E}(a))=0$ for each $a \in \mathcal{A}_{0}$. By $\tau$-maximality of $\mathcal{A}$ we have $b \in \mathcal{A}$. Thus $\mathcal{A}=\mathcal{A}_{p}$. 
Proposition 2.6. Let $0<p<1$, and let $\mathcal{A}$ be a tracial subalgebra of $\mathcal{M}$. If $\mathcal{A}$ has $L^{p_{0}}$-factorization, and $\mathcal{A}_{p_{0}}$ is a tracial subalgebra of $\mathcal{M}$ for some $0<p_{0}<1$, then

$$
\|\mathcal{E}(a)\|_{p} \leq\|a\|_{p} \quad \forall a \in \mathcal{A} .
$$

Consequently, $\mathcal{E}$ extends to a contractive projection from $[\mathcal{A}]_{p}$ onto $L^{p}(\mathcal{D})$. The extension will still be denoted by $\mathcal{E}$.

Proof. Since $\mathcal{A}_{p_{0}}$ is a subdiagonal subalgebra of $\mathcal{M}$, Theorem 2.1 of [2] implies that

$$
\|\mathcal{E}(a)\|_{p} \leq\|a\|_{p} \quad \forall a \in \mathcal{A}_{p_{0}} .
$$

From this follows (2.1).

Corollary 2.7. With the same assumption as in Proposition 2.6, we have that $\mathcal{E}(a b)=\mathcal{E}(a) \mathcal{E}(b)$ for $a \in[\mathcal{A}]_{p}$ and $b \in[\mathcal{A}]_{q}$ for $0<p, q \leq \infty$.

Proof. Note that $a b \in[\mathcal{A}]_{r}$ for any $a \in[\mathcal{A}]_{p}$ and $b \in[\mathcal{A}]_{q}$, where $1 / r=1 / p+$ $1 / q$. Thus $\mathcal{E}(a b)$ is well defined. Then the corollary follows immediately from the multiplicativity of $\mathcal{E}$ on $\mathcal{A}$ and (2.1).

\section{3. $\quad L^{2}$-DENSITY}

Definition 3.1. We say that a tracial subalgebra $\mathcal{A}$ of $\mathcal{M}$ satisfies $L^{2}$-density if $\mathcal{A}+J(\mathcal{A})$ is dense in $L^{2}(\mathcal{M})$ in the usual Hilbert space norm on that space.

It is clear that $\mathcal{A}=\mathcal{A}_{0}+\mathcal{D}, \mathcal{A}+J(\mathcal{A})=\mathcal{A}_{0}+\mathcal{D}+J\left(\mathcal{A}_{0}\right),[\mathcal{A}]_{2}=\left[\mathcal{A}_{0}\right]_{2}+[\mathcal{D}]_{2}$, and

$$
[\mathcal{A}+J(\mathcal{A})]_{2}=\left[\mathcal{A}_{0}\right]_{2}+[\mathcal{D}]_{2}+\left[J\left(\mathcal{A}_{0}\right)\right]_{2} .
$$

Hence, if $\mathcal{A}$ is an $L^{2}$-dense tracial subalgebra of $\mathcal{M}$, then we have

$$
L^{2}(\mathcal{M})=\left[\mathcal{A}_{0}\right]_{2} \oplus L^{2}(\mathcal{D}) \oplus\left[J\left(\mathcal{A}_{0}\right)\right]_{2} .
$$

Lemma 3.2. Let $\mathcal{A}$ be a $\tau$-maximal tracial subalgebra of $\mathcal{M}$ that satisfies $L^{2}$ density. Then we have

(i) $\mathcal{A}=\left(\left[J\left(\mathcal{A}_{0}\right)\right]_{1}\right)^{\perp}, \quad \mathcal{A}_{0}=\left([J(\mathcal{A})]_{1}\right)^{\perp}$;

(ii) $[\mathcal{A}]_{1}=\perp J\left(\mathcal{A}_{0}\right), \quad\left[\mathcal{A}_{0}\right]_{1}=\perp J(\mathcal{A})$;

(iii) $[\mathcal{A}]_{2}=L^{2}(\mathcal{M}) \cap[\mathcal{A}]_{1}, \quad\left[\mathcal{A}_{0}\right]_{2}=L^{2}(\mathcal{M}) \cap\left[\mathcal{A}_{0}\right]_{1}$.

Proof. (i) Assume $x \in \mathcal{A}$. If $y^{*} \in\left[J\left(\mathcal{A}_{0}\right)\right]_{1}$, then there exist $\left\{a_{n}\right\} \subset \mathcal{A}_{0}$ such that $a_{n} \rightarrow y$ in $L^{1}(\mathcal{M})$. Hence, $\tau(x y)=\lim _{n \rightarrow \infty} \tau\left(x a_{n}\right)=0$, so $\mathcal{A} \subset\left(\left[J\left(\mathcal{A}_{0}\right)\right]_{1}\right)^{\perp}$. Conversely, we take $x \in \mathcal{M}, x \in\left(\left[J\left(\mathcal{A}_{0}\right)\right]_{1}\right)^{\perp}$; i.e., $\tau(x a)=0$ for all $a$ in $\mathcal{A}_{0}$. Since $\mathcal{A}$ is $\tau$-maximal, $x \in \mathcal{A}$. Similarly, we have $\mathcal{A}_{0}=\left([J(\mathcal{A})]_{1}\right)^{\perp}$.

(ii) It is clear that $[\mathcal{A}]_{1} \subset{ }^{\perp} J\left(\mathcal{A}_{0}\right)$. Let $x \in L^{1}(\mathcal{M})$ and $x \in{ }^{\perp} J\left(\mathcal{A}_{0}\right)$. Assume $x \notin[\mathcal{A}]_{1}$. Then there exists a $y \in \mathcal{M}$ such that $\tau\left(y^{*} x\right)=1$ and $y \in\left([\mathcal{A}]_{1}\right)^{\perp}$. By (i) we obtain that $y \in J\left(\mathcal{A}_{0}\right)$. Hence, $\tau\left(y^{*} x\right)=0$, and this is a contradiction. Similarly we can prove $\left[\mathcal{A}_{0}\right]_{1}=\perp J(\mathcal{A})$.

(iii) It is obvious that $[\mathcal{A}]_{2} \subseteq L^{2}(\mathcal{M}) \cap[\mathcal{A}]_{1}$. To prove the converse inclusion, let $a \in L^{2}(\mathcal{M}) \cap[\mathcal{A}]_{1}$. Then $a \perp J\left(\mathcal{A}_{0}\right)$. From this it follows that $a \perp[J(\mathcal{A})]_{2}$. By (3.1), $a \in[\mathcal{A}]_{2} .\left[\mathcal{A}_{0}\right]_{2}=L^{2}(\mathcal{M}) \cap\left[\mathcal{A}_{0}\right]_{1}$ follows from the continuity of $\mathcal{E}$ on $[\mathcal{A}]_{2}$.

We say that $\xi \in L^{2}(\mathcal{M})$ is a right wandering vector if $\tau\left(\xi^{*} \xi b\right)=0$ for all $b \in \mathcal{A}_{0}$.

Lemma 3.3. Let $\mathcal{A}$ be a $\tau$-maximal tracial subalgebra of $\mathcal{M}$ that satisfies $L^{2}$ density. If $|\xi| \in L^{2}(\mathcal{D})$ for all right wandering vectors $\xi$, then $\mathcal{A}$ has $L^{2}$-factorization. 
The proof of Lemma 3.3 is the same as that of Theorem 3.1 of 2 . We omit the details and refer the reader to [2].

Theorem 3.4. Let $\mathcal{A}$ be a $\tau$-maximal tracial subalgebra of $\mathcal{M}$ that satisfies $L^{2}$ density. Then the following conditions are equivalent:

(i) If $x \in[\mathcal{A}]_{1}^{+}$, then $x^{\frac{1}{2}} \in H^{2}(\mathcal{A})$.

(ii) If $\xi$ is right wandering vector, then $|\xi| \in L^{2}(\mathcal{D})$.

(iii) $\mathcal{A}$ is a subdiagonal subalgebra of $\mathcal{M}$.

Proof. (ii) $\Rightarrow$ (iii) follows from Lemma 3.3 and Theorem 2.4

(iii) $\Rightarrow$ (i) is trivial.

(i) $\Rightarrow$ (ii). Let $\xi$ be a right wandering vector. Then

$$
\tau\left(\xi^{*} \xi b\right)=0, \forall b \in \mathcal{A}_{0} .
$$

By (ii) of Lemma 3.2 we have $\xi^{*} \xi \in[\mathcal{A}]_{1}$, and $|\xi| \in[\mathcal{A}]_{2}$. Hence,

$$
\tau(|\xi| b)=0, \forall b \in \mathcal{A}_{0}+J\left(\mathcal{A}_{0}\right)
$$

So by the $L^{2}$-density of $\mathcal{A}$, it follows that $|\xi| \in L^{2}(\mathcal{D})$.

Theorem 3.5. Let $\mathcal{A}$ be a tracial subalgebra of $\mathcal{M}$ that satisfies $L^{2}$-density. Then the following conditions are equivalent:

(i) $\mathcal{A}$ is a subdiagonal subalgebra of $\mathcal{M}$.

(ii) For some $0<p<1, \mathcal{A}$ has $L^{p}$-factorization and $\mathcal{A}_{p}$ is a tracial subalgebra of $\mathcal{M}$.

Proof. (ii) $\Rightarrow\left(\right.$ i). Since $\mathcal{A}_{p}$ is a subdiagonal subalgebra of $\mathcal{M}$,

$$
L^{2}(\mathcal{M})=\left[\mathcal{A}_{p}\right]_{2} \oplus\left[J\left(\left(\mathcal{A}_{p}\right)_{0}\right)\right]_{2} .
$$

So from

$$
L^{2}(\mathcal{M})=[\mathcal{A}]_{2} \oplus\left[J\left(\mathcal{A}_{0}\right)\right]_{2},[\mathcal{A}]_{2} \subset\left[\mathcal{A}_{p}\right]_{2},\left[J\left(\mathcal{A}_{0}\right)\right]_{2} \subset\left[J\left(\left(\mathcal{A}_{p}\right)_{0}\right)\right]_{2},
$$

it follows that $[\mathcal{A}]_{2}=\left[\mathcal{A}_{p}\right]_{2}$. If $x \in L^{2}(\mathcal{M})^{-1}$, then there is a unitary $u \in \mathcal{M}$ and $a \in\left[\mathcal{A}_{p}\right]_{2}^{-1}=[\mathcal{A}]_{2}^{-1}$ such that $x=u a$, since $\mathcal{A}_{p}$ is a subdiagonal subalgebra. Hence, $\mathcal{A}$ has $L^{2}$-factorization. By Theorem 2.4 we obtain the desired result.

\section{REFERENCES}

[1] W. B. Arveson, Analyticity in operator algebras, Amer. J. Math 89 (1967), 578-642. MR0223899 (36:6946)

[2] T. N. Bekjan, Q. Xu, Riesz and Szegö type factorizations for noncommutative Hardy spaces, J. Operator Theory 62 (2009), 215-231. MR2520548

[3] D. P. Blecher, L. E. Labuschagne, Logmodularity and isometries of operator algebras, Trans. Amer. Math. Soc. 355 (2003), 1621-1646. MR1946408 (2004c:46113)

[4] D. P. Blecher, L. E. Labuschagne, Characterization of noncommutaive $\mathcal{H}^{\infty}$, Integr. Equ. Oper. Theory 56 (2006), 301-321. MR 2270840 (2007m:46101)

[5] D. P. Blecher, L. E. Labuschagne, Applications of Fuglede-Kadison determinant: Szegö's theorem and outers for noncommutative $H^{p}$, Trans. Amer. Math. Soc. 360 (2008), 61316147. MR2425707 (2009m:46096)

[6] R. Exel, Maximal subdiagonal algebras, Amer. J. Math 110 (1988), 775-782. MR955297 (90b:46114)

[7] T. Fack, H. Kosaki, Generalized s-numbers of $\tau$-measurable operators, Pac. J. Math 123 (1986), 269-300. MR840845 (87h:46122)

[8] G. Ji, K.-S. Saito, Factorization in subdiagonal algebras, J. Funct. Anal. 159 (1998), 191-202. MR:1654186 (99k:46106) 
[9] S. Kawamura, J. Tomiyana, On subdiagonal algebras associated with flows in operator algebras, J. Math. Soc. Japan 29 (1977), 73-90. MR0454650 (56:12899)

[10] L. E. Labuschagne, A noncommutative Szegö theorem for subdiagonal subalgebras of von Neumann algebras. Proc. Amer. Math. Soc. 133 (2005), 3643-3646. MR2163602 (2006d:46081)

[11] M. Marsalli, Noncommutative $H^{2}$-spaces, Proc. Amer. Math. Soc. 125 (1997), 779-784. MR.1350954 (97e:46089)

[12] M. Marsalli, G. West, Noncommutative $H^{p}$-spaces, J. Operator Theory 40 (1997), 339-355. MR 1660390 (2000b:46117)

[13] M. Marsalli, G. West, The dual of noncommutative $H^{1}$, Indiana Univ. Math. J. 47 (1998), 489-500. MR.1647920 (99h:46122)

[14] M. McAsey, P. S. Muhly, K.-S. Saito, Nonselfadjoint crossed products (invariant subspaces and maximality), Trans. Amer. Math. Soc. 248 (1979), 381-409. MR.522266 (80j:46101b)

[15] G. Pisier, Q. Xu, Non-commutative $L^{p}$-spaces, in Handbook of the geometry of Banach spaces, Vol. 2, North-Holland, Amsterdam, 2003, 1459-1517. MR.1999201(2004i:46095)

[16] N. Randrianantoanina, Hilbert transform associated with finite maximal subdiagonal algebras, J. Austral. Math. Soc. (Series A) 65 (1999),388-404. MR 1660424 (2000a:46109)

[17] K.-S. Saito, On non-commutative Hardy spaces associated with flows on finite von Neumann algebras, Tohoku Math. J. 29 (1977), 585-595. MR0463932 (57:3870)

[18] K.-S. Saito, A note on invariant subspaces for maximal subdiagonal algebras, Proc. Amer. Math. Soc. 77 (1979), 349-352. MR545594 (81b:46078)

[19] M. Takesaki, Duality for crossed products and the structure of von Neumann algebras of type III, Acta Math. 131 (1973), 249-310. MR0438149 (55:11068)

[20] M. Terp, $L^{p}$-spaces associated with an arbitrary von Neumann algebra, Notes, Math. Institute, Copenhagen Univ., 1981.

College of Mathematics and Systems Sciences, Xinjiang University, Urumqi 830046, People's Republic of China 